\title{
Meteorological Visibility Evaluation on Webcam Weather Image Using Deep Learning Features
}

\author{
Shengyan Li, Hong Fu, and Wai-Lun Lo
}

\begin{abstract}
The estimation methods of meteorological visibility currently in use on digital image are mainly based on the meteorological laws and the corresponding features are extracted manually to calculate the visibility. However, besides the parameters of camera setting, the environmental and weather conditions will also affect the image quality and cause different kinds of noise to influence the evaluation accuracy. It is hard to extract all these factors manually and involve them into a certain equation to solve the visibility. Therefore, it is necessary to intelligently extract the useful factors and evaluate visibility. In this paper, an intelligent digital method is proposed to estimate the visibility on webcam weather image. In this method, a pre-trained convolutional neural network (CNN) is employed to automatically extract the visibility features instead of manual method and a generalized regression neural network (GRNN) is designed for intelligent visibility evaluation based on these deep learning features. A series of weather photo with the ground truth of visibility is used to train and test the proposed method. The result shows that the proposed automatic method with deep learning feature is workable for visibility prediction, whose accuracy is higher than that of the traditional method with hand-crafted features.
\end{abstract}

Index Terms-Meteorological visibility, weather photo, deep learning, feature extraction.

\section{INTRODUCTION}

Meteorological visibility is a measure of the transparency of the atmosphere, which is an important factor for road, air and water transportation safety. The pollution problem also increases the need of visibility measurement. As many factors, such as dust, smoke, fog and haze, will influence the visibility, its measurement and estimation is still a very complex problem. The visibility monitoring mainly includes visual observation by trained meteorological observers, optical measurement by visibility meter and digital evaluation on camera image. In visual method, human being can generally ignore the noise caused by different weather condition and make a visual estimation of meteorological optical range (MOR) by recognizing the natural and man-made targets at the greatest distance. So the visual method depends on the number of reference targets in the observation environment and the contrast determination based on observer's vision. Limited to human feeling, the visibility observation can just be conducted hourly, that is quite a long time for the visibility verification. Besides, with city development, the tall buildings are also

Manuscript received April 5, 2017; revised October 23, 2017.

All authors are with the Department of Computer Science, Chu Hai College of Higher Education, Hong Kong (e-mail: syli@chuhai.edu.hk, hfu@chuhai.edu.hk, wllo@chuhai.edu.hk). harmful to visual method. Currently, the visibility meter is the most popular method for automatic visibility measurement. Optical sensors measure the light extinction to estimate meteorological optical range, which is defined as the length of atmosphere over which a beam of light travels before its luminous flux is reduced to $5 \%$ of its original value. The visibility meter includes transmission method, forward-scatter method and backscatter method. With respects to sensitivity, correctness, compactness and price, forward-scatter meter is the most widely used in automatic visibility measurement, which measures a small portion of light scattered out of a light beam for the estimation of extinction coefficient. The equipment is very expensive and needs high installation requirements. It can just measure a short path of the atmosphere, so the accuracy is limited.

Compared to the visibility meters, camera-based visibility monitoring techniques are close to the visual method and have been widely used to estimate the visibility for decades [1]-[15]. The camera-based method can be mainly divided into three classes: one is used for road transportation based on road camera [1]-[5]. In these works, the special references on road, such as the straight road or the road marks, are needed to calculate the meteorological optical range. As the road camera just contains the road scene within a short distance, these methods are used for low visibility on road. The second image method is reference method, corresponding to visual method. For example, in the work of Bäumer et al. [6], 39 visible targets are provided in the panorama and the visibility is evaluated by detecting whether or not the edge of targets is visible. Enough targets are needed for the measurement range and accuracy in this method. The third image method is according to the law in meteorology, such as Koschmieder equation or Lambert-beer law [7]-[15]. In this method, features related to the extinction coefficient or visibility distance are extracted from the image to evaluate the visibility based on the meteorological law. For example, Babari et al. [7]-[9] proposed a physics-based model to express the mapping between the contrast and the atmospheric visibility distance with the consideration of Lambertian surfaces of the scene. Carretas et al. [10] used two tree sets located at different but known distances to calculate the extinction coefficient based on the different wavelengths associated with $\mathrm{R}, \mathrm{G}, \mathrm{B}$ channels of the image to get the visibility. In digital image method, the image quality and reference targets are both important for feature extraction and visibility evaluation. The image quality, such as the image contrast, is determined by many factors, including light source (the sun), weather condition and camera setting. It is hard to extract all the related 
features to describe the complex effect of the factors on visibility.

In this paper, an intelligent digital method based on webcam image will be proposed to estimate meteorological visibility by two neural networks. One is a pre-trained $\mathrm{CNN}$ for automatic feature extraction, which can extract thousands of features directly from image. Then according to the correlation coefficient between the features and the visibility, the related features can be selected as input of the another neural network, a generalized regression neural network (GRNN), for function approximation of visibility. In the rest of this paper, the visibility image database will be constructed and described in Section II. The measurement law of meteorological visibility will be briefly introduced in Section III and the algorithm of intelligent visibility estimation will be also proposed in this section. Some discussions, such as the comparison of the effects of the proposed deep learning feature and the hand-crafted feature on visibility evaluation, and the effect of image size, will be presented in Section IV and V. Finally, a conclusion will be given in Section VI.

\section{Database Construction OF VisibiLity IMAGE}

The webcam-based weather photos of Tsim Sha Tsui and the corresponding visibility are provided by the Hong Kong Observation (HKO) as the visibility image database for this paper.

\section{A. Visibility Report}

The Hong Kong Observation measures the visibility in Hong Kong Waters at five weather stations: (1) Hong Kong Observatory, (2) Chek Lap Kok, (3) Central, (4) Sai Wan Ho and (5) Waglan Island, and provides these visibility reports on their website [16]. Fig. 1 shows an example image of the visibility reports. In these weather stations, the visibility at Hong Kong Observatory is based on hourly visual observations by a trained meteorological observer and the others are derived from automatic visibility readings of visibility meter.

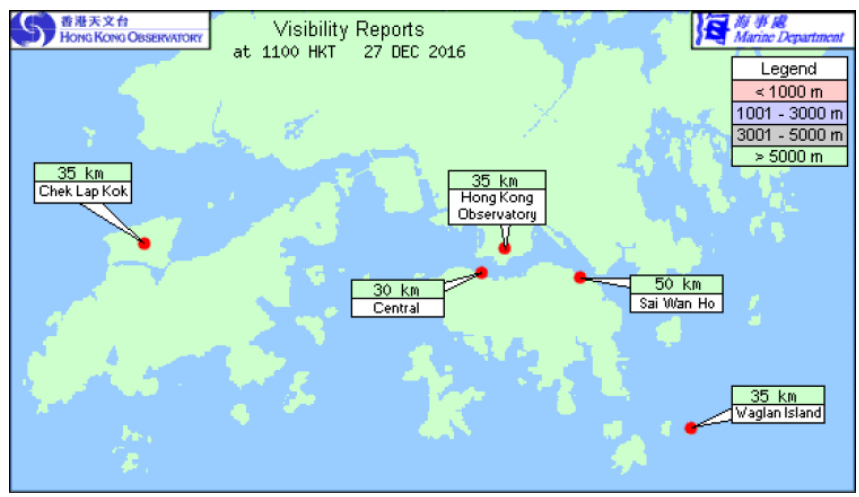

Fig. 1. Visibility readings in Hong Kong Waters [16].

\section{B. Weather Photo by Webcam}

The Hong Kong Observation also sets up 24 webcams which distribution is shown in Fig. 2(a), to automatically take weather photos in every 5 minutes and the photos are also released to public on their website [16]. The visibility evaluation by image method is similar to that by visual method. Refer to Fig. 1, we know that the visibility of weather photo taken by webcam No. 10 is corresponding to the visibility visually observed at Hong Kong Observation. Hence, the visibility monitored by visual method at Hong Kong Observatory is considered as the ground truth for the weather photo obtained by the webcam No.10. Fig. 2(b) shows an example of the weather photo taken by webcam No. 10, which contains the scene of Tsim Sha Tsui looking towards the west. Its landmarks are marked in the photo shown in Fig. 2(c) for reference.

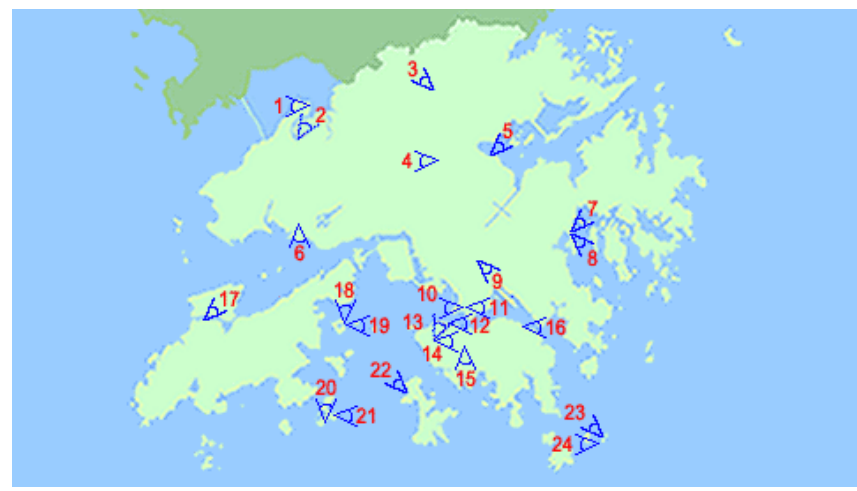

(a) Webcam distribution of $\mathrm{HKO}$

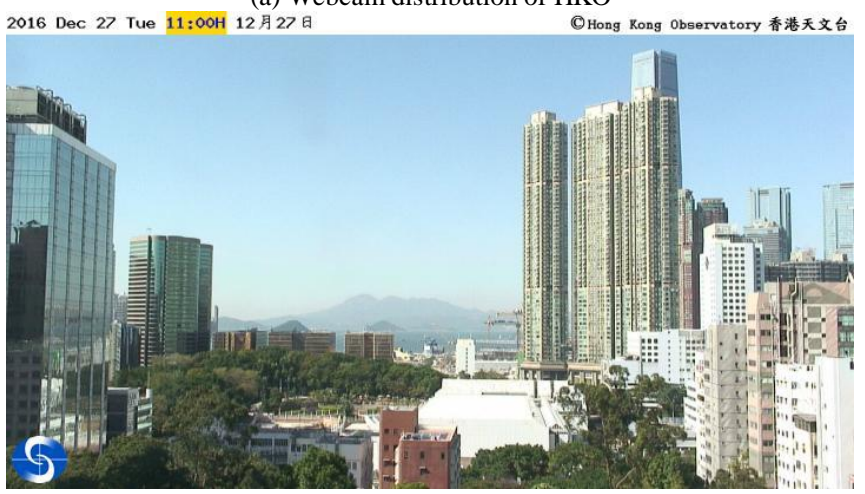

(b) Weather photo obtained by webcam No. 10

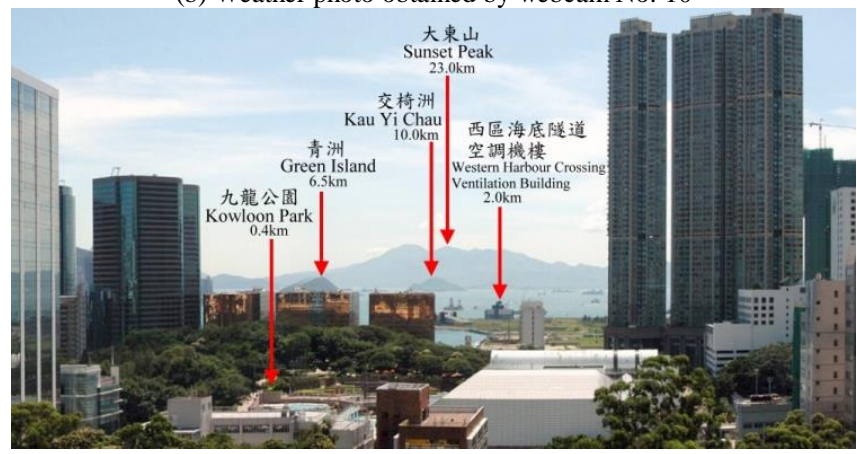

(c) Landmark of weather photo in (b)

Fig. 2. Webcams of HKO and weather photo [17]

\section{Visibility Image Database}

Based on above, we collect the day-time weather photo taken by webcam No.10 and its visibility reading of Hong Kong Observation from Oct 2016 to Feb 2017 to build up the visibility image database for this paper. As the webcams are installed outdoors, the environmental factor is possible to affect the image quality. In this work, if the photo is blurred due to the heavy rain water, the weather photo will be deleted 
from the database. The scene in the camera is a part of the view of the meteorological observer (like a $360^{\circ}$ panoramic image) and the distribution of atmospheric particulate concentrations may not be uniform. So, some weather photos are deleted from the database if the visibility in the report is significantly differentiated from the visual value of the photo. Finally, a visibility image database is constructed by 1003 weather photos with the visibility reading at daytime $(7 \mathrm{am} \sim 5 \mathrm{pm})$. The distribution of visibility in the database is shown in Fig. 3. The visibility range in this database is from $5 \mathrm{~km}$ to $35 \mathrm{~km}$. The visibility sample in $26 \mathrm{~km}, 28 \mathrm{~km}$ and $29 \mathrm{~km}$ is missing.

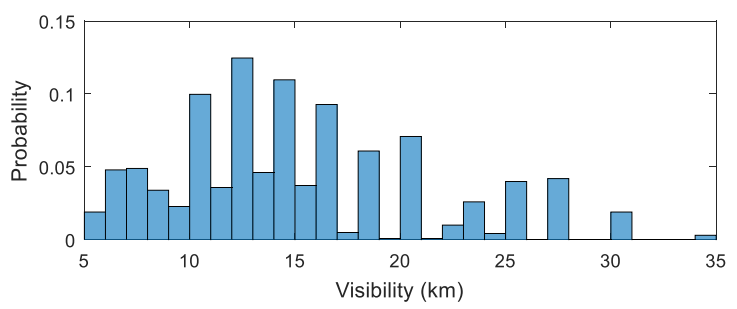

Fig. 3. Visibility distribution of the image database.

\section{Methodology}

Traditionally, the visibility is estimated by the theory of the apparent luminance of objects against background sky on the horizon, named Koschmieder equation. In this equation, a relationship between the apparent luminance $I$ of an object and the extinction coefficient of the atmosphere $k$ is established as:

$$
I=I_{0} e^{-k d}+I_{b}\left(1-e^{-k d}\right)
$$

where $I_{b}$ is the luminance of the sky; $I_{0}$ and $d$ are the intrinsic luminance and the distance of the object, respectively.

Then Lambert-beer law is further proposed to estimate the extinction coefficient, which is an attenuation law of atmospheric contrasts:

$$
C=C_{0} e^{-k d}
$$

where $C$ and $C_{0}$ are the apparent contrast at distance $d$ and the intrinsic contrast of the object against its background.

The contrast between the object and the background can be written as

$$
C=\frac{I_{b}-I}{I_{b}}
$$

The International Commission on Illumination (CIE) has adopted a contrast threshold of 0.05 to define the meteorological visibility distance $d_{v i s}$. So if the object is black $\left(C_{0}=1\right), d_{v i s}$ can be obtained by

$$
d_{v i s}=-\frac{1}{k} \ln (0.05) \cong \frac{3}{k}
$$

According to above equations, uniform illumination of the atmosphere, clearness of sky or background, intrinsic luminance of the target, the precise of target distance, the luminance of image, the cloud, the location of sun, dust, smoke, fog, haze and so on, will influence the accuracy of visibility estimation. It is very complicated to extract the suitable features to express so much information. Hence, deep learning is employed in this study to intelligently extract the related features for visibility estimation. The procedure for the proposed method of visibility evaluation is shown in Fig. 4. In the proposed method, the original weather photo will be firstly processed to reduce noise and as input of a pre-trained CNN. Then, thousands of features will be automatically extracted from the image. Followed that, the related deep learning features will be selected as input of a GRNN to approximate the function of visibility.

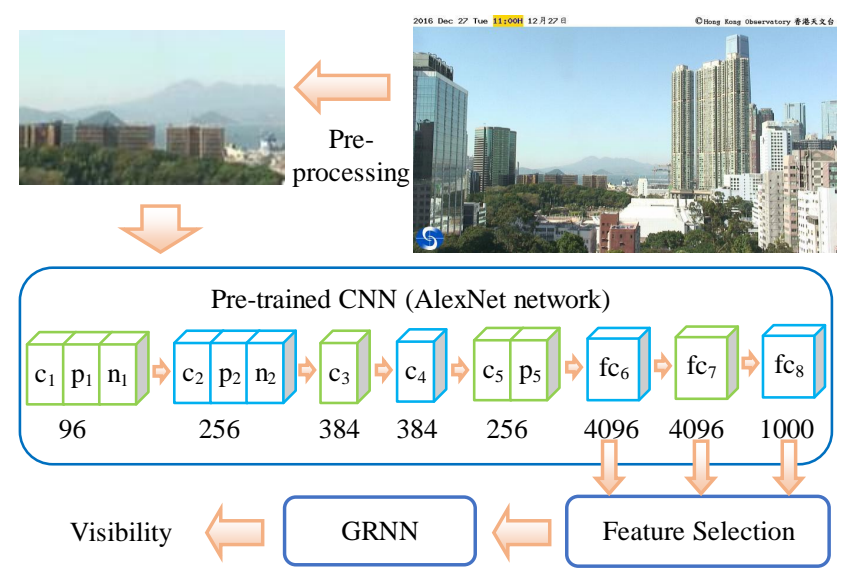

Fig. 4. Procedure for visibility estimation. $c_{i}$ is convolutional layer; $p_{i}$ is pooling layer; $\mathrm{n}_{\mathrm{i}}$ is local response normalization and $\mathrm{fc}_{\mathrm{i}}$ is fully connected layer.

\section{A. Image Processing}

Although the webcam is fixed, the weather photos still have a small movement in the long-term image acquisition, due to the shaking in image capture or equipment maintenance. In this paper, the image registration based on speeded up robust features (SURF) is applied to get a static image series. Then a $2 \times 2$ Gaussian filter is used on the webcam image to reduce noise. According to the landmark in Fig. 2(c), the original weather photo shown in Fig. 2(b), seems to contain many tall buildings, which are close to the webcam and are the obstacle for the distant scene. These should be the redundant information and may not be useful for visibility estimation. So here just the central region of the weather photo with the size of 110×190, as shown in Fig. 5, is kept for further processing.

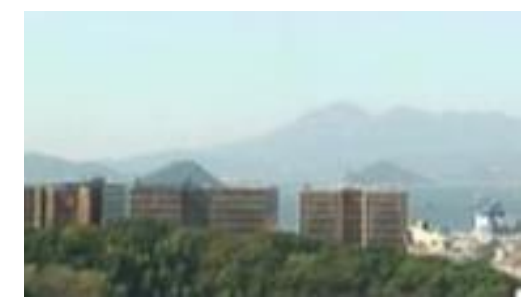

Fig. 5. Input weather image with visibility of $35 \mathrm{~km}$. 


\section{B. Feature Extraction by pre-Trained Model}

$\mathrm{CNN}$ can learn the useful representations of features directly from input image. The accuracy of deep learning model largely depends on the amount of training dataset. Usually, it requires thousands or even millions of samples to train a $\mathrm{CNN}$, because it needs to learn millions of weights. In this paper, there is about one thousand samples in the database. It is not enough to train a new CNN. Transfer learning is an alternative way without the need for a huge data set, which can use a pre-trained deep learning model to extract features from a new data set automatically. In this study, a pre-trained model named AlexNet network [18] is employed to extract the visibility features automatically. The pre-trained AlexNet network has 60 million parameters and 650000 neurons, consists of five convolutional layers, some of which are followed by max-pooling layers, and three fully-connected layers with a final 1000-way softmax [19]. As shown in Fig. 4, the features in the three fully-connected layers are all extracted for feature selection. So total number of the extracted features is 10812, including 4906 features from the layer of fc6, 4906 features from the layer of fc7 and 1000 features from the layer of fc8.

In feature selection, the correlation coefficient between these 10812 features and the visibility is calculated to select the high-related features. In this study, the features with the correlation coefficient higher than 0.4 , will be selected. In this stage, 330 features from fc6, 308 features from fc7 and 162 features from fc8, are selected from the 10812 features obtained by the pre-trained AlexNet network. Then a stepwise regression model is used to further select the significant features by a multilinear model based on their statistical significance in a regression. The critical $p$ value of the $F$-statistic sets as 0.005 . Finally, 10 features from fc6 layer and 8 features from fc7 layer are selected for visibility estimation. Fig. 6 shows the correlation coefficient of the selected features and visibility.

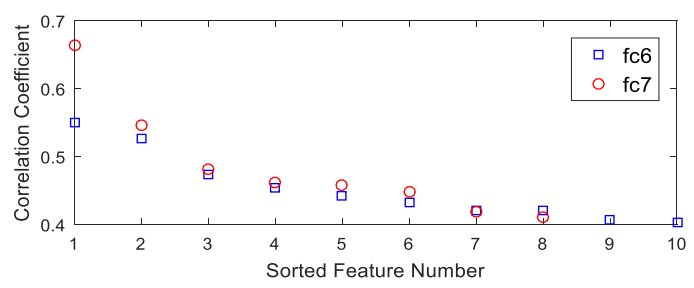

Fig. 6. Correlation coefficient between features and visibility.

\section{Visibility Evaluation}

In this study, a GRNN is designed to estimate visibility. GRNN is a kind of four-layer radial basis network, which consists of an input layer, a radial basis layer, a special linear layer and an output layer. Based on above, total 18 features are extracted and selected from the visibility image, so the designed GRNN contains 18 inputs and 1 output of visibility. In GRNN, the biases of radial basis layer are set as $0.8326 /$ spread. In this paper, the spread is searched by mean absolute error (MAE):

$$
\mathrm{MAE}=\frac{1}{n} \sum_{i=1}^{n}\left|e_{i}\right|=\frac{1}{n} \sum_{i=1}^{n}\left|s_{i}-y_{i}\right|
$$

where $n$ is the number of testing sample, $e_{i}$ is the absolute error, $s_{i}$ is the predicted visibility and $y_{i}$ is the real visibility.

We define $70 \%$ of the data as training dataset and $30 \%$ of the data as testing dataset. So, there is 702 data in training dataset and 301 data in testing dataset. The visibility distribution of training and testing dataset is shown in Fig. 7.
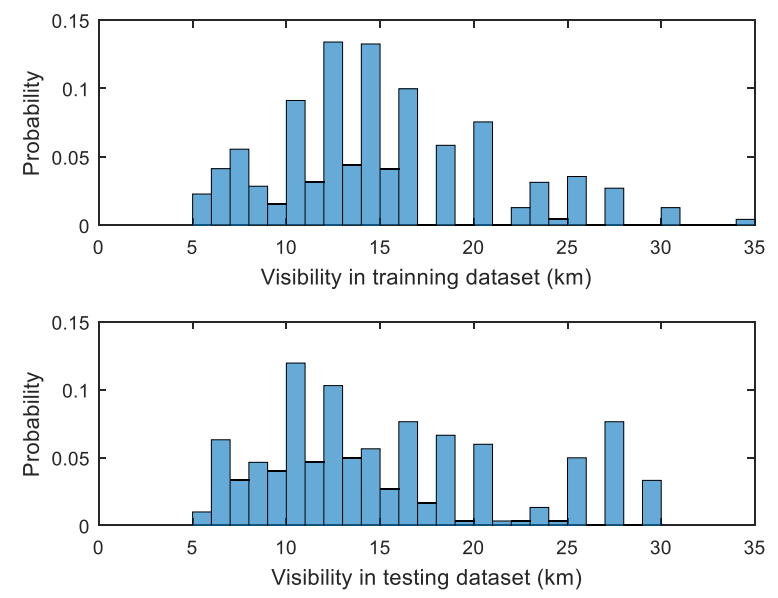

Fig. 7. Visibility distribution of training and testing dataset.

According to (5), the relationship between the spread and the mean absolute error of testing data is calculated and shown in Fig. 8. In order to obtain minimum mean absolute error, the spread is set as 0.45 .

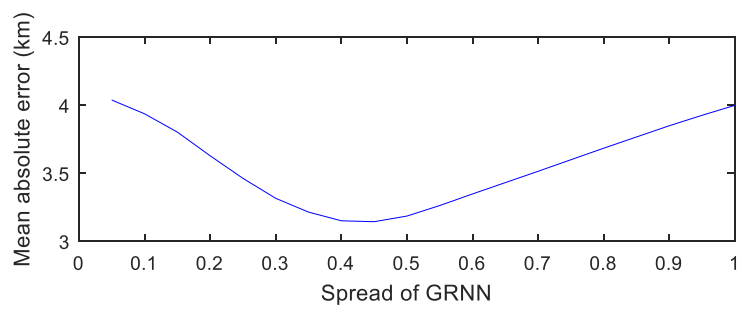

Fig. 8. Spread and mean absolute error.

Therefore, the GRNN with 18 inputs and 1 output for visibility prediction is obtained by training the 702 data with the spread of 0.45 . Then the trained network is used to predict the visibility of the training dataset and testing dataset, respectively. The result is shown in Fig. 9.
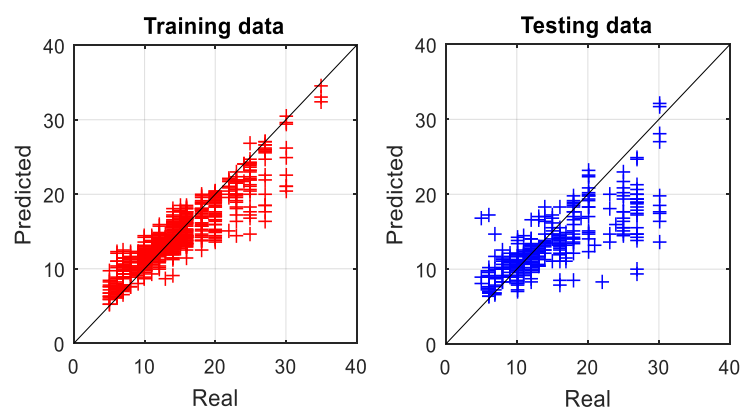

Fig. 9. Simulation results.

We define that if the predicted visibility is within the $20 \%$ 
of the real visibility, prediction is accurate. From Fig. 9, the result shows that about $61.8 \%$ of testing samples can be correctly predicted and accuracy of training dataset is about $77.9 \%$. In order to analyze the simulation results, the histogram of the error of training and testing data is shown in Fig. 10. The predicted error is concentrated in $\pm 3 \mathrm{~km}$.
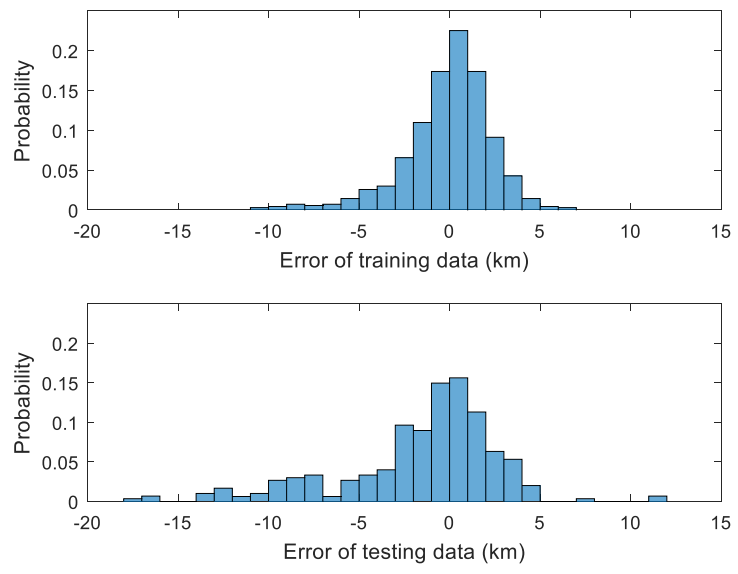

Fig. 10. Histogram of training and testing error.

\section{COMPARISON WITH HAND-CRAFTED FEATURES}

The accuracy of the proposed method which is based on deep learning features, is about $61.8 \%$. In this section, the hand-crafted features will be extracted according to meteorological laws for visibility evaluation to compare with the proposed method.

\section{A. Contrast Feature}

As mentioned before, the definition of visibility is related to image contrast. The contrast of the low-visibility image should be lower. Here, Michelson contrast is expressed as feature $f_{1}$ :

$$
f_{1}=\frac{I_{\text {max }}-I_{\text {min }}}{I_{\text {max }}+I_{\text {min }}}
$$

where $I_{\max }$ and $I_{\min }$ are the maximum and minimum of image luminance, respectively.

Fig. 11 shows the gray image of Fig. 5. According to landmarks in Fig. 2(c), the $2 \times 2$ regions: $A_{b}, A_{1}, A_{2}$ and $A_{3}$ in Fig. 11, represent the background of sky and the landmarks of mountain targets 1 3 (Sunset Peak, Kau Yi Chau and Green Island). The distances between the mountain targets $1 \sim 3$ and the camera are $23 \mathrm{~km}, 10 \mathrm{~km}$ and $6.5 \mathrm{~km}$, respectively. The mean intensities $I_{b}, I_{1}, I_{2}$ and $I_{3}$ of $A_{b}, A_{1}, A_{2}$ and $A_{3}$ are considered as the intensities of sky and mountain targets 1 3.

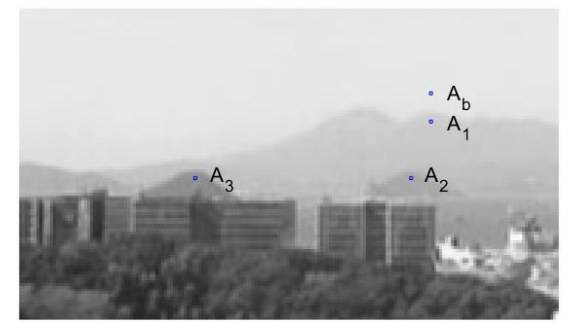

Fig. 11. Gray image and regions: $A_{b}, A_{1}, A_{2}$ and $A_{3}$.
The contrast between the mountain targets and the background can be obtained by

$$
C_{i}=\frac{I_{i}-I_{b}}{I_{b}}, i=1 \sim 3
$$

If $C_{i}$ is larger than $5 \%$, the $i^{\text {th }}$ mountain target is visible. However, the intensity of sky may be influenced by haze, cloud and sunshine, $C_{i}$ is a relative value. Suppose the mountains have same intrinsic intensity in same image, the contrast $g_{i}$ among mountains should be more comparative:

$$
g_{i}=\frac{I_{i}-I_{i-1}}{I_{i-1}}, i=2 \sim 3
$$

So, we define the anther contrast feature $f_{2}$ as

$$
f_{2}=\left(1+C_{1}\right) \prod_{i=2}^{3}\left(1+g_{i}\right)
$$

where if $C_{1}$ and $\mathrm{g}_{2}$ is less than $5 \%$, let them equal to zero.

\section{B. Transmission Feature}

Dark channel prior [20] can be used to calculate relative transmission map from a single image based on (1). Fig. 12 shows the transmission map $t(x)$ of Fig. 11. The transmission $t(x)$ is expressed as

$$
t(x)=e^{-k d(x)}
$$

where $d(x)$ is the distance between $x$ and camera.

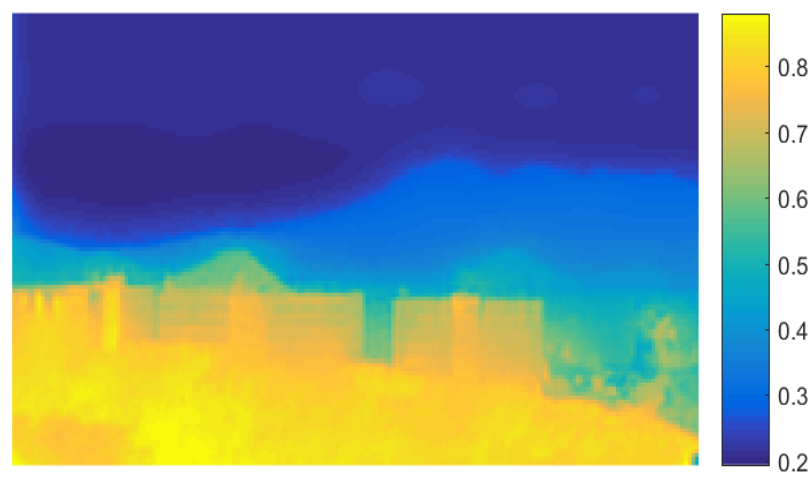

Fig. 12. Transmission image of Fig. 11.

The scattering coefficient of the atmosphere can be obtained by

$$
k=-\frac{1}{\sum_{i=1}^{1} \alpha_{i}} \sum_{i=1}^{3}\left(\alpha_{i} \frac{\ln \left(t_{i}\right)}{d_{i}}\right)
$$

where $t_{i}$ and $d_{i}$ are the transmission and distance of $i^{\text {th }}$ mountain target in Fig. 11; $\alpha_{i}$ is equal to zero when the intensity contrast of target $C_{i}$ or $g_{i}$ to the background is less than 5\%; otherwise $\alpha_{i}$ is equal to 1 .

Then the visibility distance $d_{v i s}$ can be obtained by (4), as 
feature $f_{3}$.

\section{Gradient Feature}

With the decrease of visibility, the scene in the image becomes less and blurry, so the gradient of the image should express some characteristics of the images in different visibilities. In this study, the gradient image is obtained by Sobel operator, and the sample image is shown in Fig. 13. The mean of gradient image is considered as feature $f_{4}$.

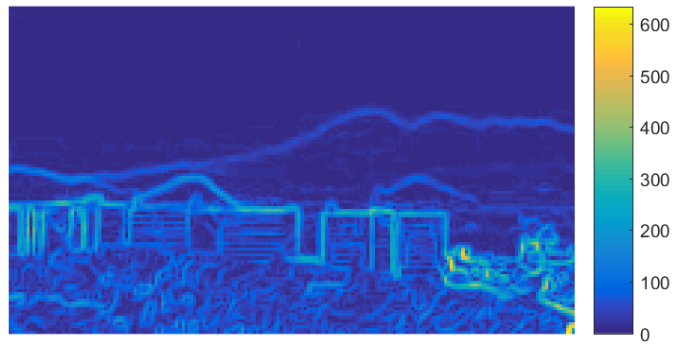

Fig. 13. Gradient image of Fig. 11.

\section{Visibility Evaluation}

With the extracted hand-crafted features $f=\left[f_{1} f_{2} f_{3} f_{4}\right]$ for each visibility image, the GRNN with four inputs and one output is trained by 702 data in training dataset with the spread of 0.20 , to predict the visibility. The prediction results of training dataset and testing dataset are shown as Fig. 14. The corresponding errors of training and testing dataset are shown in Fig. 15. The accuracy of the training dataset and the testing dataset is $63.1 \%$ and $56.8 \%$, which is lower than those of the proposed method.
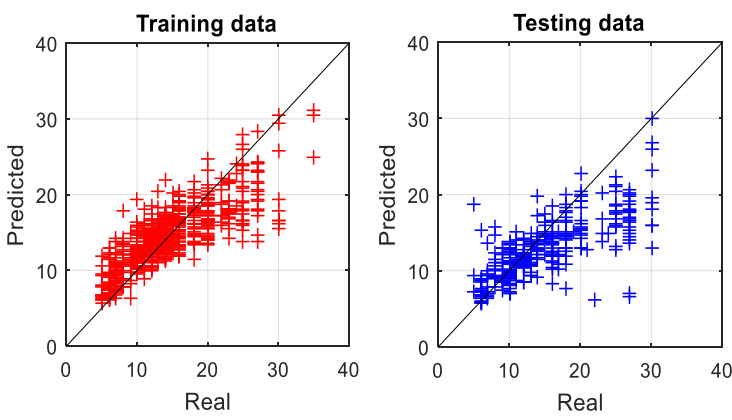

Fig. 14. Scatter plot of real and predicted visibility (km).
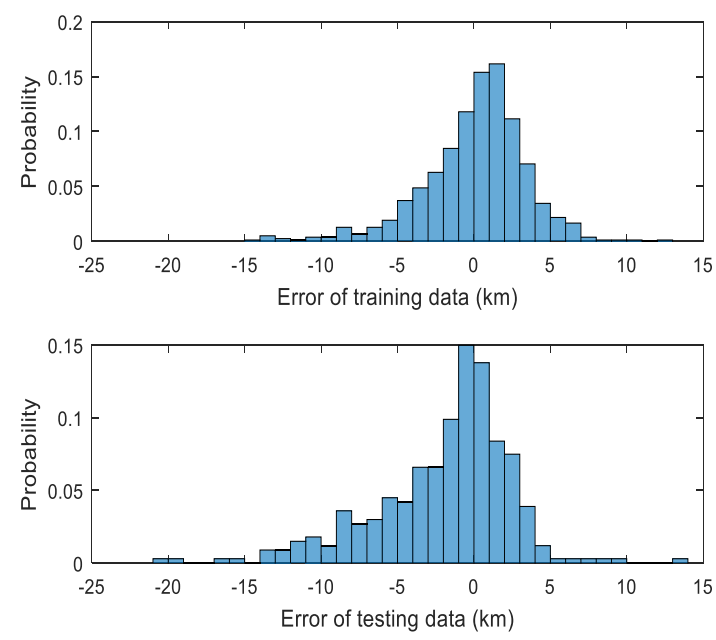

Fig. 15. Histogram of the error of predicted results.
In this section, it is noted that the factors and features for representative of visibility in real day is complex due to the environmental and weather condition. The hand-crafted feature is also hard to describe the visibility although we spend a lot time to design it. For different scenes, the hand-crafted features will be much different. Compared to hand-crafted feature, the proposed method based on deep learning feature is much helpful in visibility evaluation, which is an automatic method and higher accuracy is obtained for visibility prediction. It should be easier to extend to predict the visibility for different scenes.

\section{EFFECT OF IMAGE SIZE}

In this paper, we cut a part of the image as input for the pre-trained $\mathrm{CNN}$ to extract features for visibility evaluation. In this section, the effect of region size on the visibility estimation will be discussed. As shown in Fig. 16, different regions of image are designed to cut as input: (a) Case 1: 380×620; (b) Case 2: 277×277; (c) Case 3: 110×190; (d) Case 4: $60 \times 190$. Case 3 is the sample size in the proposed method and the details are described in Section III.

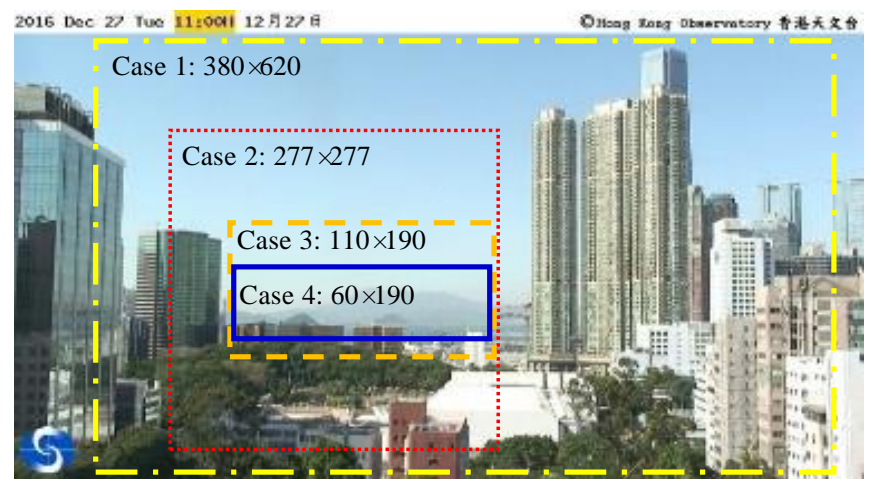

Fig. 16. Different size of weather photo for evaluation.

Followed the algorithm in Section III, the comparison on image size is listed in Table I. It is noted that context of the image will influence the accuracy of the visibility prediction. With the decrease of image size, the ratio of context which is changed significantly in different visibility, is increased and the accuracy for visibility prediction also increases. If the context of the image is high-variance region for the visibility, the accuracy will be the highest. It reveals that the context of image in proposed method will influence the measurement range and accuracy of visibility.

TABLE I: THE COMPARISON ON IMAGE SIZE

\begin{tabular}{lllll}
\hline \hline & Case 1 & Case 2 & Case 3 & Case 4 \\
& $380 \times 620$ & $277 \times 277$ & $110 \times 190$ & $60 \times 190$ \\
\hline No of input feature & 29 & 23 & 18 & 21 \\
Spread & 0.6 & 0.5 & 0.45 & 0.5 \\
Training accuracy & 72.8 & 76.1 & 77.9 & 79.6 \\
Testing accuracy & 54.8 & 53.2 & 61.8 & 61.8 \\
SAE $(\mathrm{km})$ & 3.665 & 3.713 & 3.143 & 3.198 \\
\hline \hline
\end{tabular}


Due to the limitation of image resolution of webcam, the available region is a small part of the image with a low image resolution, which is even less than the size of input image for the pre-trained $\mathrm{CNN}$ model $(277 \times 277)$. This will cause the noise to be enlarged. So, this makes a suggestion that the parameter setting of the webcam: the available region should be larger than or close to the size of input image.

\section{CONCLUSION}

In this paper, an intelligent digital method was proposed to estimate the visibility of webcam image based on two neural networks. The CNN is used for feature extraction and GRNN is designed for function approximation. The proposed algorithm is applied on the weather photo of Tsim Sha Tsui and predicted accuracy can reach to about $62 \%$, which is higher than the method based on hand-crafted features (57\%). If the percentage of effective region in the weather photo is higher, the prediction accuracy will also be higher. In the future, the proposed method should be applied to the image obtained by other weather stations to test the accuracy on different scenes, and it can be also compared with the visibility measured by automatic visibility meter and used as an automatic visibility detection algorithm.

\section{ACKNOWLEDGMENT}

The work described in this paper was fully supported by a grant from the Research Grants Council of the Hong Kong Special Administrative Region, China (Project Reference No.: UGC/IDS13/14). The authors would also like to thank Hong Kong Observatory for the providing the weather photos and visibility reports as database.

\section{REFERENCES}

[1] N. Hautiére, J. P. Tarel, J. Lavenant, and D. Aubert, "Automatic fog detection and estimation of visibility distance through use of an onboard camera," Machine Vision and Applications, vol. 17, no. 1, pp. 8-20, 2006.

[2] N. Hautière, D. Aubert, É. Dumont, and J. P. Tarel, "Experimental validation of dedicated methods to in-vehicle estimation of atmospheric visibility distance," IEEE Transactions on Instrumentation and Measurement, vol. 57, no. 10, pp. 2218-2225, 2008.

[3] N. Hautière and A. Boubezoul, "Combination of roadside and in-vehicle sensors for extensive visibility range monitoring," in Proc. Sixth IEEE International Conference on Advanced Video and Signal Based Surveillance (AVSS'09), IEEE, September 2009, pp. 388-393.

[4] Z. Z. Chen, J. Li, and Q. M. Chen, "Real-time video detection of road visibility conditions," in Proc. 2009 WRI World Congress on Computer Science and Information Engineering, March 2009, IEEE, vol. 5, pp. $472-476$.

[5] M. Negru and S. Nedevschi, "Image based fog detection and visibility estimation for driving assistance systems," in Proc. 2013 IEEE International Conference on Intelligent Computer Communication and Processing (ICCP), IEEE, pp. 163-168, September 2013.

[6] D. Bäumer, S. Versick, and B. Vogel, "Determination of the visibility using a digital panorama camera," Atmospheric Environment, vol. 42, no. 11, pp. 2593-2602, 2008.

[7] R. Babari, N. Hautiere, E. Dumont, R. Brémond, and N. Paparoditis, "A model-driven approach to estimate atmospheric visibility with ordinary cameras," Atmospheric Environment, vol. 45, no. 30, pp. 5316-5324, 2011.

[8] R. Babari, N. Hautière, É. Dumont, N. Paparoditis, and J. Misener, "Visibility monitoring using conventional roadside cameras-Emerging applications," Transportation Research Part C: Emerging Technologies, vol. 22, pp. 17-28, 2012.

[9] R. Babari, N. Hautière, E. Dumont, J. P. Papelard, and N. Paparoditis, "Computer vision for the remote sensing of atmospheric visibility," in Proc. 2011 IEEE International Conference on Computer Vision Workshops (ICCV Workshops), IEEE, November 2011, pp. 219-226

[10] F. Carretas, F. Wagner, and F. M. Janeiro, "Atmospheric visibility and Angström exponent measurements through digital photography," Measurement, vol. 64, pp. 147-156, 2015.

[11] K. W. Kim, "Estimation of visibility using a visual image," Environmental Monitoring and Assessment, vol. 187, no. 3, pp. 1-10, 2015.

[12] J. Wang, X. Liu, X. Yang, M. Lei, S. Ruan, K. Nie, Y. Miao, and J. Liu, "Development and evaluation of a new digital photography visiometer system for automated visibility observation," Atmospheric Environment, vol. 87, pp. 19-25, 2014.

[13] T. Hagiwara, S. Fujita, and K. Kizaka, "Assessment of visibility on roads under snow conditions using digital images," in Proc. 11th International Road Weather Conference, January 2002.

[14] L. Xie, A. Chiu, and S. Newsam, "Estimating atmospheric visibility using general-purpose cameras," in Proc. International Symposium on Visual Computing, Springer Berlin Heidelberg, December 2008, pp. 356-367.

[15] J. P. Andreu, S. Mayer, K. Gutjahr, and H. Ganster, "Measuring visibility using atmospheric transmission and digital surface model," arXiv preprint arXiv:1505.05286, 2015.

[16] Visibility readings in Hong Kong Waters. [Online]. Available: http://www.hko.gov.hk/vis/vis_index.shtml

[17] Regional Weather in Hong Kong. [Online]. Available: http://www.hko.gov.hk/wxinfo/ts/index_webcam_e.htm

[18] Pretrained models. [Online]. Available: http://www.vlfeat.org/matconvnet/pretrained/

[19] A. Krizhevsky, I. Sutskever, and G. E. Hinton, "Imagenet classification with deep convolutional neural networks," in Proc. Advances in Neural Information Processing Systems, 2012, pp. 1097-1105.

[20] K. He, J. Sun, and X. Tang, "Single image haze removal using dark channel prior," IEEE Transactions on Pattern Analysis and Machine Intelligence, vol. 33, no. 12, pp. 2341-2353, 2011.

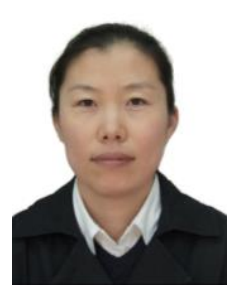

Shengyan Li received her Ph.D. degree in the Institute of Textiles and Clothing from the Hong Kong Polytechnic University in 2016.

Her research interests include image processing, computer vision, eye tracking techniques and numerical analysis.

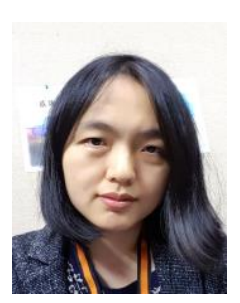

Hong $\mathrm{Fu}$ received her bachelor and master degrees from Xi'an Jiao tong University in 2000 and 2003, and Ph.D. degree from the Hong Kong Polytechnic University in 2007.

She is now an associate professor in the Department of Computer Science, Chu Hai College of Higher Education, Hong Kong. Her research interests include eye tracking, computer vision, pattern recognition, and artificial intelligence.

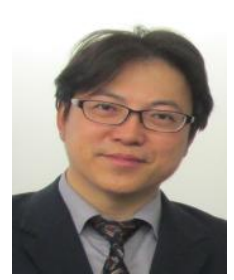

Wai-Lun Lo received the B.Eng. degree in electrical engineering and the Ph.D. degree from the Hong Kong Polytechnic University, Kowloon, in 1991 and 1996, respectively.

$\mathrm{He}$ is currently the head of the Department and professor of Computer Science, Chu Hai College of Higher Education. His research interest includes adaptive control, fuzzy systems, application of machine learning in power electronics systems. 